\title{
Ueber Psyllostearylalkohol.
}

Von

Ernst Fdw. Sundwik.

(Der Redaction zugegangen am 6. Mărz 1898.)

Zweite Mittheilung.

Vor mehreren Jahren (diese Zeitschrift, Jahrgang 1893, Seite 425) habe ich eine vorläufige Mittheilung gemacht über einen Alkohol von der Zusammensetzung $\mathrm{C}_{33} \mathrm{H}_{66} \mathrm{O}$, welchen ich in der von Psylla Alni secernirten fettartigen Substanz entdeckt habe. Ich hatte diese Substanz mit concentrirter Bromwasserstoffsäure (Spec. Gew. 1,49) bei $210^{\circ}$ bis $220^{\circ} \mathrm{C}$. behandelt und dabei gefunden, dass der Stoff möglicher Weise in ein Bromid übergeht. - Die einfachste Formel der ursprünglichen Substanz war also $\mathrm{C}_{33} \mathrm{H}_{66} \mathrm{O}$. Als Alkohol musste derselbe ein ungesättigter sein.

In den folgenden Jahren habe ich stets nur ungenügende Mengen von der Substanz erhalten und deshalb meine Untersuchung nur langsam fördern können. Es ist aber möglich gewesen, die Zusammerisetzung und das Molekulargewicht der Verbindung festzustellen, und es stimmen diese neueren Resultate nicht völlig mit denjenigen der ursprünglichen Mittheilung überein.

Wie schon angegeben, ist die ursprüngliche Substanz in kaltem Alkohol, Aether und Chloroform fast unlöslich, auch unlöslich in heissem Aether. In heissem Chloroform ist der Stoff leicht löslich, auch ziemlich leicht löslich in heissem Alkohol, wenn er nur vorher wasserfrei gemacht wurde. Der Stoff ist schwer angreifbar. Schmelzen mit pulverförmigem 
Kalihydrat und sehr wenig Alkohol haben keine Einwirkung. Nachdem ich nochmals die Aufschliessung mit Bromwasserstoffsäure vorgenommen hatte, bemerkte ich bald, dass der Gehalt an Brom in dem Reactionsprodukte stark variirte. Es zeigte sich zuletzt, dass ein mit Wasser gut behandeltes Produkt völlig bromfrei war, ebenso dass die Krystallform unverändert blieb auch nach dem Behandeln des Reactionsproduktes mit Natriumamalgam und wasserhaltigem Alkohol. Hierdurch ist erwiesen, dass der ursprüngliche Stoff ebenso wie das Reactionsprodukt gesättigte Verbindungen sind, und dies ist um so sicherer, als sie beide in Chloroformlösung kein Brom aufnehmen.

Ich hatte schon früher einige Molekulargewichtsbestimmungen mit dem ursprünglichem Stoffe nach Beckmann's chemischer Methode gemacht, wobei Chloroform als Lösungsmittel diente. Dabei ergab sich Folgendes:

\begin{tabular}{|c|c|c|c|c|c|c|}
\hline Versuch & No. & & rgab & als & Molekulargewicht & 954,6 \\
\hline$»$ & $»$ & II. & $»$ & $\gg$ & $»$ & 1070,0 \\
\hline$\triangleright$ & » & III. & $\triangleright$ & 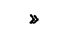 & 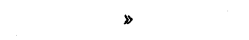 & 841,0 \\
\hline ه & » & IV. & 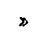 & » & » & 1072,0 \\
\hline , & » & V. & * & 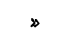 & 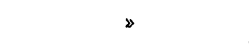 & 984,5 \\
\hline
\end{tabular}

Die Formel $\mathrm{C}_{33} \mathrm{H}_{66} \mathrm{O}$ muss also verdoppelt und als Molekül $\mathrm{C}_{66} \mathrm{H}_{132} \mathrm{O}_{2}=956$ angenommen werden, was auch mit den folgenden Untersuchungen völlig übereinstimmt.

Das mit Bromwasserstoffsäure (Spez. Gew. 1,49) bei $210^{\circ}-220^{\circ}$ während dreiviertel Stunde erhaltene Produkt krystallisirte aus Benzol in schönen 4seitigen (bisweilen 6seitigen) Tafeln, deren Winkel durch Ocular-Goniometer gemessen wurden. Als Mittel zahlreicher Messungen ergaben sich die Winkel zu $74^{\circ}$ und $106^{\circ}$. Wie schon früher angeführt worden, krystallisirt der ursprüngliche Stoff in Nadeln.

Der Stoff schmilzt (nach vorhergegangener Austreibung des Krystallwassers durch Erhitzen über $100^{\circ}$ G.) bei $87^{\circ}$ bis $88^{\circ}$ C., die ursprüngliche Substanz bei $96^{\circ}$ C. - Das neue Produkt besitzt in hohem Grade die Fähigkeit, Wasser zu binden, wie die folgenden Analysen zeigen. - Der ursprüngliche Stoff 
besitzt auch diese Eigenschaft in nicht geringerem Grade, wie ich durch viele frühere Untersuchungen festgestellt habe. In Folge dessen müssen beide Stoffe, um wasserfrei zu werden, entweder Monate hindurch über Schwefelsäure im Vacuum gehalten, oder auch über $100^{\circ}$ C. erhitzt werden.

Ich habe schon früher das Reactionsprodukt nach Behandlung mit Bromwasserstoffsäure analysirt und verschiedene Resultate erhalten, welche schwer mit den übrigen Befunden in Zusammenhang $\mathrm{zu}$ bringen waren. Nun werden diese Verhältnisse klar. Es zeigte sich bei der mikroskopischen Untersuchung, ebenso beim Umkrystallisiren, mit grösster Wahrscheinlichkeit, dass sich nur ein Körper bildete. Dieser gab als einfachste Formel bei der Analyse $\mathrm{C}_{11} \mathrm{H}_{29} \mathrm{O}_{12}$ (sehr nahe) und $\mathrm{C}_{33} \mathrm{H}_{68} \mathrm{O}_{2}$, welche Formel später als demselben Stoffe zugehörend gefunden worden. Das Wasser bedingt den Unterschied. Während des Aufbewahrens hatte sich zuletzt alles Wasser verflüchtigt; daher sind die Zahlen der späteren Analyse zu erklären.

Um zu zeigen, wie der ursprüngliche Stoff mit Wasser sich verbindet, führe ich hier nur eine Analyse an.

Die Substanz war aus Chloroform auskrystallisirt und hatte eine Nacht über Schwefelsäure im Vacuum gestanden.

$$
\begin{array}{r}
\text { I. } 0,2188 \text { gr. gab } 0,5495 \mathrm{CO}_{2} \text { oder } 68,59 \% \mathrm{C}_{2} \\
0,2188 \text { \% } 0,2688 \mathrm{H}_{2} \mathrm{O} \text { \% } 13,656 \% \mathrm{H}_{2} \\
\mathrm{C}_{66} \mathrm{H}_{132} \mathrm{O}_{2} 11 \mathrm{H}_{2} \mathrm{O} \text { erfordern } 68,63 \% \mathrm{C}_{2} \text { und } 13,34 \% \mathrm{H}_{2} .
\end{array}
$$

Die wasserfreie Substanz ist, wie schon in voriger Mittheilung angegeben, mehrmals mit ganz übereinstimmenden Resultaten analysirt.

Das Reactionsprodukt mit Bromwassersäure.

Vor der Analyse über $100^{\circ} \mathrm{C}$. erhitzt.

II. 0,182 gr. gab $0,5342 \mathrm{CO}_{2}$ oder $80,05 \% \mathrm{C}_{2}$ $0,182 \gg 0,2252 \mathrm{H}_{2} \mathrm{O} \gg 13,75 \% \mathrm{H}_{2}$

$\mathrm{C}_{33} \mathrm{H}_{66}<\underset{\mathrm{OH}}{\mathrm{OH}}$ erfordert $79,84 \% \mathrm{C}_{2}$ und $13,70 \% \mathrm{H}_{2}$.

III. Die aus Benzol krystallisirte Substanz war eine Nacht über Schwefelsäure in Vacuum gestanden.

0,1694 gr. gab $0,3646 \mathrm{CO}_{2}$ oder $58,701 \% \mathrm{C}_{2}$

0,1694 » $0,2030 \mathrm{H}_{2} \mathrm{O}$ » $13,312 \% \mathrm{H}_{2}$ 
Hieraus berechnet sich die Formel: $\mathrm{C}_{33} \mathrm{H}_{66}<{ }_{\mathrm{OH}}^{\mathrm{OH}} 10 \mathrm{H}_{2} \mathrm{O}$, welche erfordert: $58,58 \% \mathrm{C}_{2}$ und $13,02 \% \mathrm{H}_{2}$. Hier sind also etwas weniger als $10 \mathrm{H}_{2} \mathrm{O}$.

IV. Das Produkt, zweimal aus Petroläther umkrystallisirt, 2 Nächte über Schwefelsäure bei theilweise evacuirter Luft gestanden.

0,1324 gr. gab $0,2612 \mathrm{CO}_{2}$ oder $53,81 \% \mathrm{C}_{2}$ $0,1324 \gg 0,15466 \mathrm{H}_{8} \mathrm{O} \gg 12,98 \% \mathrm{H}_{2}$

Die Formel $\mathrm{C}_{33} \mathrm{H}_{66}<{ }_{\mathrm{OH}} \mathrm{OH} 13 \mathrm{H}_{2} \mathrm{O}$ erfordert $54,02 \% \mathrm{C}_{2}$ und $12,88 \% \mathrm{H}_{2}$.

V. 2 Monate über Schwefelsäure unter Glasglocke gestandenes Produkt, wobei die grösste Menge Wasser abgegangen ist.

$$
\begin{aligned}
& 0,1687 \text { gr. gab } 0,4473 \mathrm{CO}_{2}=72,312 \% \mathrm{C}_{2} \\
& 0,1687 \text { ه } 0,2039 \mathrm{H}_{8} \mathrm{O}=13,423 \% \mathrm{H}_{2} .
\end{aligned}
$$

Die Formel $\mathrm{C}_{33} \mathrm{H}_{66}<\mathrm{OH}_{3} 3 \mathrm{H}_{2} \mathrm{O}$ erfordert $72,00 \% \mathrm{C}_{2}$ und $13,46 \% \mathrm{H}_{2}$. Es waren also nicht mehr völlig 3 Moleküle $\mathrm{H}_{2} \mathrm{O}$ zurückgeblieben.

Es zeigen diese Analysen also, dass der Stoff wenigstens 13. Moleküle $\mathrm{H}_{2} \mathrm{O} \mathrm{zu}$ binden vermág, und dass dieses Wasser nur langsam abgegeben wird. Auch kann es keinem Zweifel unterliegen, dass die durch Bromwasserstoffsäure bewirkte Zersetzung in folgender Weise zugegangen ist:

$$
\mathrm{C}_{33} \mathrm{H}_{66}<{ }_{0}^{0}>\mathrm{C}_{38} \mathrm{H}_{66}+\mathrm{H}_{2} \mathrm{O}=\mathrm{C}_{33} \mathrm{H}_{66}<\underset{\mathrm{OH}}{\mathrm{OH}}+{ }_{\mathrm{HO}}^{\mathrm{HO}}>\mathrm{C}_{33} \mathrm{H}_{66}
$$

Die Alkoholnatur des Reactionsprodukts mit Bromwasserstoffsäure habe ich nicht nur durch die Analyse und die Zersetzung selbst, sondern auch durch die gewöhnlichen Alkoholreactionen bestätigen können. Auch der Schmelzpunkt und mehrere andere Eigenschaften sprechen dafür, dass ein zweiwerthiger Alkohol hier entstehen muss und entstanden ist.

Auf diesen Alkohol übertrage ich den früher von mir angewandten Namen Psyllostearylalkohol.

Sein Schmelzpunkt ist $86^{\circ}-87^{\circ}$ C. (uncorr.). Er krystallisirt (mit Wasser) in schrägen, kantenförmigen Tafeln, aus Essigester einmal in dicken Prismen, und ist, wasserhaltig, unlöslich oder sehr schwer löslich in Aether, sehr schwer löslich in Petroläther von niedrigerem Kochpunkt, leicht in Benzol und Essigester. Im wasserfreien Zustand löst er sich 
leicht in kochendem Aether, auch in Alkohol, und in den übrigen genannten Lösungsmitteln auch gewöhnlich sehr leicht.

Wie man sieht, enthält die Substanz grosse Mengen Wasser, von welchem sie durch vorhergegangenes Schmelzen und Erhitzen, beziehungsweise längeren Aufenthalt im Vacuum frei gemacht werden kann. Ich will aber nicht behaupten, dass gerade 13 Moleküle $\mathrm{H}_{2} \mathrm{O}$ der grösste Gehalt sei, sondern es ist mir sogar wahrscheinlich, dass noch einige Moleküle mehr darin vorhanden sein können. - Ein anderes Verhalten ist aber nicht weniger bemerkenswerth. Auch die ursprüngliche Substanz, der Aether $\mathrm{C}_{33} \mathrm{H}_{66}<_{0}^{0}>\mathrm{C}_{33} \mathrm{H}_{66}$, nimmt in gleicher Weise Wasser auf, wie es mehrere Analysen, die ich hier nicht anführe, gezeigt haben. Diese grosse Wassermenge hatten mich Anfangs ganz irre geführt, ehe ich vor der Verbrennung den Stoff $\mathrm{zu}$ schmelzen und bis $110^{\circ}$ bis $120^{\circ} \mathrm{C}$. zu erhitzen begann, wie ich schon früher angegeben habe.

In biologischer Hinsicht wird gewiss diese Wasserbindung von grosser Bedeutung sein, besonders wenn man bedenkt, wie relativ rasch und leicht unter günstigen Bedingungen alles Wasser abgegeben wird, und ebenso wie leicht das Wasser wieder bei feuchter Witterung aufgenommen wird. Denn einerseits sind die Thiere in der Nacht dem feuchtkalten Thau ausgesetzt, andererseits haben sie die austrocknende Wirkung der Mittagssonne zu ertragen. Schon die Fettdecke, welche von dem Aether gebildet wird, ist ein gutes Schutzmittel gegen Wärmestrahlung und Regen. Mir ist es sehr wahrscheinlich, dass die in dieser Weise ausgerüsteten Insecten, die ihre Wohnstätte nur schwer und selten wechseln können, durch Wasserabgabe am Tage und durch Wasseraufnahme bei feuchter Witterung, z. B. in der Nacht, die Eigenwärme in gewissem Grade reguliren könnten: durch Wärmebindung in der Nacht, durch Wärmeabgabe bei Tage. Beides wird ermöglicht durch den Wechsel der Wassermenge in der schützenden Hülle. Auch die chemische Bindung des Wassers, die vorwiegend Nachts erfolgt, muss zu einer Erwärmung führen. Der ursprüngliche Stoff verhält sich nichts- 
destoweniger wie Fette, indem er nicht von Wasser benetzt wird - ein geeignetes Schutzmittel beim Regen. Die gegen die kleinen Zweige des Baumes gedrückte untere Seite des Thieres braucht ein solches Schutzmittel nicht. Man versteht leicht, warum die Thiere nach einem stärkeren Regen zum grössten Theil vernichtet werden. Sie sind überhaupt, auch wenn man das genannte Secret in Rechnung nimmt, sehr schlecht gegen äussere Einflüsse geschützt. Ohne die Schutzmittel trocknet ein Individuum in der Sonne sehr bald und schrumpft ein.

Helsingfors, Physiologisch-chemisches Institut, den 26. Februar 1898. 